Historic, Archive Document

Do not assume content reflects current scientific knowledge, policies, or practices. 


$$
\text { . }
$$




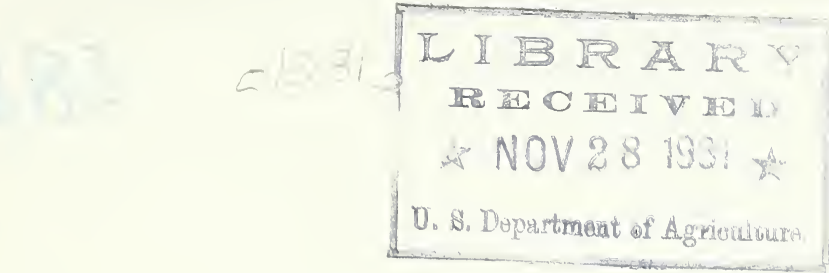

\section{RARE PLANTS AND BULBS}

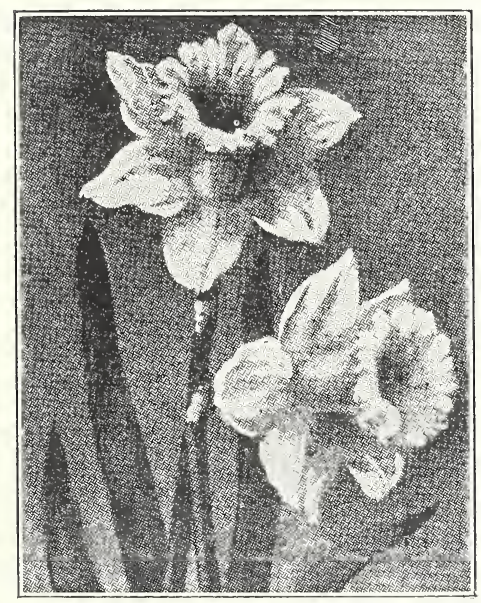

\section{Rosalie M. Dumble Davis \\ Originator and Grower of}

Iris, Rockplants, Roses, Narcissus and Perennials

$$
\text { tes }
$$

\section{MOSS-SIDE}

2535 MacGREGOR W A Y

HOUSTON, TEXAS 


\section{New and Rare Plants and Bulbs}

We call your attention to the following varieties:

Among the Narcissus, GARDEN GEM, a very old variety but one that is splendid for naturalizing. RED GOLD, one of the most beautiful of the Boetaz group; and INGLESIDE BEAUTY, which originated in one of our own gardens. Of these we have sufficient stock to enable us to offer them at exceedingly low rates. Among the perennials and rockplants, GEUM BORISSI is very lovely. Hemerocallis, QUEEN BESS, a volunteer in a bed of Queen of May, we are introducing this year. LYTHRUM LINEARE, besides its other good qualities, colors most beautifully in the fall. VERONICA TEXANA presented some years ago to the garden by Mother Nature, is one of the most attractive and satisfactory rockplants that we know. Among the Sedums are a number that are unusual and delightful. While our rare old roses might properly be termed "Fool-Proof Roses" as they can be moved at any time, are exceedingly hardy, and grow and bloom in weeds and grass as well as in the best kept garden. In bloom they are a joy, and no garden should be without them.

\section{SPRING FLOWERING BULBS NARCISSUS}

(C. cup or crown; T. trumpet; P. perianth) GIANT TRUMPET DAFFODILS

APRICOT. A dainty thing for the rock garden. P. white, T.

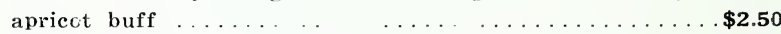

ALICE KNIGHTS. T. creamy, P. pure white ........... 25 DUKE OF BEDFORD. P. white, T. soft yellow ........ 1.00

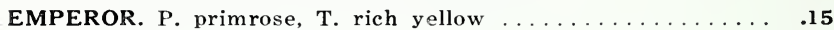

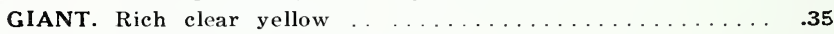

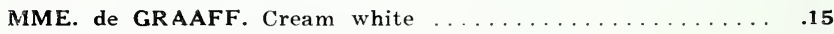

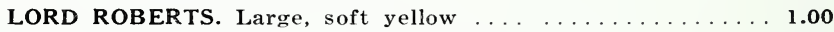
OLYMPIA. $\mathrm{P}$. prim rose, $\mathrm{T}$. slightly deeper ........... .20 SPRING GLORY. P. white, T. deep yellow ............15 VAN WAVEREN'S GIANT. Huge, rich yellow .......... 25

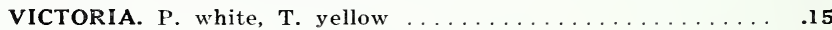

\section{NARCISSUS INCOMPARABILIS}

CROESUS. Very large flat orange C. against a creamy P... 1.50 GLORIA MUNDI. P. yellow, C. scarlet ............15 LUCIFER. P. sulphur, C. chrome edged orange . . . . . . .15

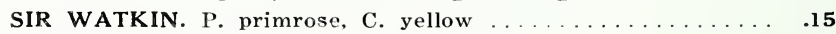

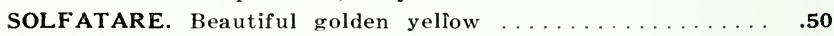
TORCH. Star shoped. P. yellow, C. orange ........... 50 WILL SCARLETT. Cream P., frilled C. scarlet ......... .50

\section{NARCISSUS BARRI}

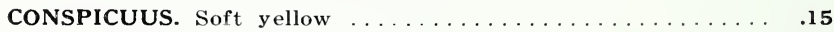

FLAME. Enormous soft yellow, C. edged crimson . . . . 35

LADY GODIVA. P. white, C. yellow, edged red ... . . . .15

RED CHIEF. P. white, fringed C. orange scarlet $\ldots \ldots \ldots \ldots . .25$

\section{NARCISSUS LEEDSII}

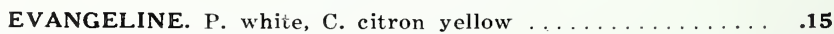

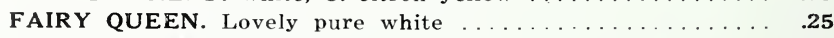

LORD KITCHENER. Very large. P. white, C. lemon ....... 50

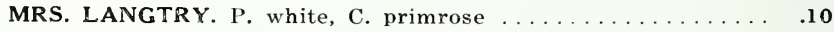


PEACH. A rock garden gem. P. white, C. peach . . . . . .\$1.00 WHITE QUEEN. Pure white. Very fine . . . . . . . 1.00

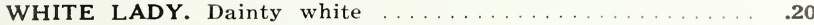

\section{NARCISSUS POETICUS}

GLORY. A huge poeticus. P. white, C. red

\section{NARCISSUS POETAZ}

GARDEN GEM. Almost a Leedsii in size and shape, bearing one to five flowers to stem; P. white, C. lemon. Very late RED GOLD. Very brilliant, P. Temon, spreading, crinkled, gold C. edged red

\section{NARCISSUS TRIDIMUS}

MRS. ALFRED PEARSON. Something different in bunch flowered narcissus. Three to five lovely, graceful, white flowers with long cream trumpet, instead of cup or crown, on one stem

\section{DOUBLE NARCISSUS}

ARGENT. White with yellow center

GOLDEN ROSE. Quite lovely. Shaped like a peony with very double center

INGLESIDE BEAUTY. Phoenix type. Pure white, with small

inner petals of vivid orange, edged deep red ....... 1.00

ALBA PLENA ODORATA. Snowy white, fragrant....... 10

\section{JONQUIL}

CAMPERNELLE. Golden yellow, fragrant

\section{MISCELLANEOUS BULBS}

Scilla, Wood Hyacinths, or Blue Bells

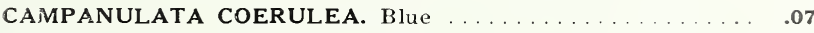

.. “ White Queen. Pure white... .08

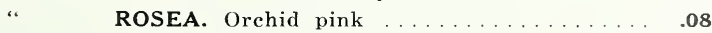

“ EXCELSIOR. Very tall. Blue ......... .08

Muscari, Grape Hyacinths

BOTRYOIDES. Naturalizes easily. Blue ............ .05

\section{Spanish Iris}

BLANCHE FLEUR. White, tinted pale blue ............10

CAJANUS. Large clear yellow . . . . . . . . . . . . . . . . .10

WHITE EXCELSIOR. Fine white .................. 10

IMPERATOR (I. FILIFOLIA) Rich deep blue ..........10

Leucojum, Snowflakes

VERNUM. Dainty, nodding white flowers .............. 10

\section{Crinum}

HYBRIDS. Pure white to dark pink

\section{Hardy Perennials and Rock Garden Plants}

\$Rock Plants.**Equally good in rockery or border.

\section{ASTER}

BARR'S PINK. Showy pink. Gold bronze center

Campanula RAPUNCULOIDES. Tall spikes of lovely lavender blue bells 
APRICOT Rich apricot .................... $\mathbf{3 0}$

AURANTIACA. Orange, shaded brown $\ldots \ldots \ldots \ldots \ldots \ldots \ldots \ldots . .30$

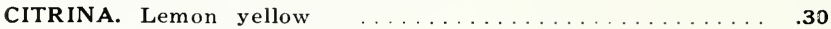

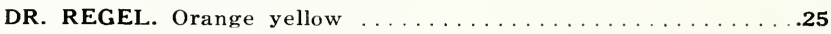

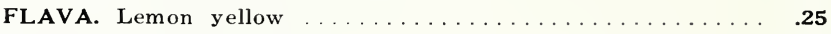

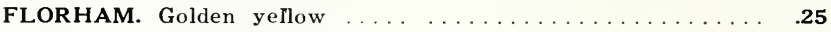

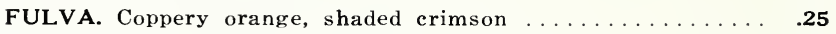

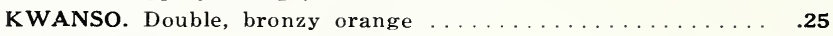

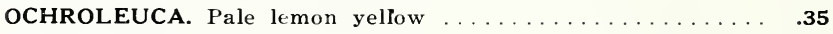

QUEEN BESS. New. Large double flowers of soft apricot,

slight stain of crimson where lemon throat begins ..... 1.50

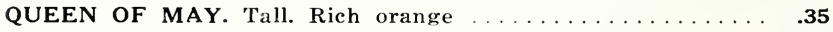

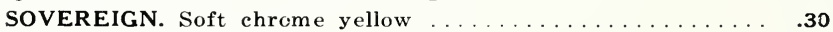

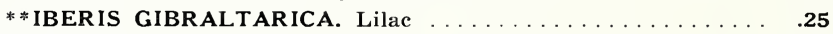

IRIS. See Iris list.

**LYTHRUM LINEARE. Native. Prostrate foliage fall and winter. Flower stems 2 to 3 feet in summer, forming graceful bush, covered with small pink lavender flowers. Good for cutting

* POLEMOMIUM HUMILE. Pale blue Early $\ldots \ldots \ldots \ldots \ldots \ldots$

PRIMULA VULGaRIS. True English Primrose $\ldots \ldots \ldots \ldots \ldots . .35$

\section{SEDUM}

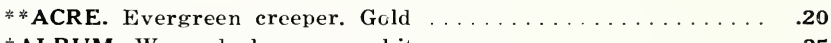

ALBUM. Waxy dark green; white . . . . 25

$\$$ BROWNII. Reddish purple in winter; blue $\ldots \ldots \ldots \ldots \ldots . .35$

**CORDIFOLIUM. Low green rosettes; rose $\ldots \ldots \ldots \ldots \ldots . .35$

†DASYPHILLUM. Bluish-grey beads; blush $\ldots \ldots \ldots \ldots \ldots . .35$

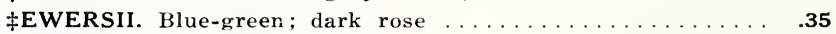

${ }^{* *}$ GLAUCUM. Light blue-green; white $\ldots \ldots \ldots \ldots \ldots \ldots \ldots .25$

¥IBERICUM. Dark green; pink or white $\ldots \ldots \ldots \ldots \ldots \ldots \ldots .35$

* JAPONICUM MACROPHYLLUM. Upright, pale green .... . 35

**JAPONICUM VARIEGATA. White and green; flesh ..... . 35

${ }^{*}$ KAMTSCHATICUM. Sage green; orange $\ldots \ldots \ldots \ldots \ldots .25$

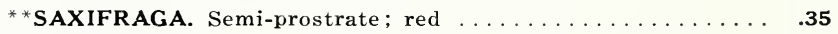

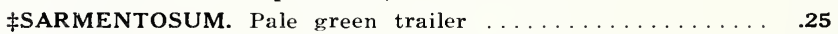

${ }^{*}$ SPECTABILE. Upright grower; rosy crimson . . . . . . 35

* SPECTABILE ALBA. Same as above; white . . . . . 35

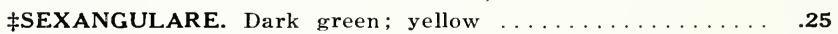

†SPURIUM COCCINEUM. Reddish green; rose . . . . . . . 25

†STELLARIS. Waxy green; reddish purple ...........35

**XL. (in doubt) greenish yellow; bronzy yellow ........ .25

**VERONICA TEXANA. Dark green cushions covered all summer and fall with tiny white stars, followed by atractive bronze seed pods. Fine for edging or rockwork. Native..

**VIOLET CUCULATA. Long Stemmed, dark blue ....... 20

${ }^{*}$ VIOLET ALBA. White veined blue .............

\section{VINES}

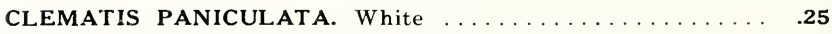

HONEYSUCKLE. Variegated foliage $\ldots \ldots \ldots \ldots \ldots \ldots \ldots \ldots . .35$

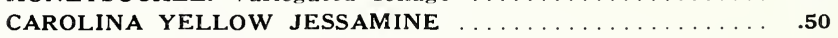

**YLLOW CREEPER. Good ground cover. Attractive foliage, dainty yellow daisy-like blooms ............35

** MORONGIA ROEMERIANA. Mimosa leaves, and adorable pink puff-ball flowers

\section{RARE OLD ROSES}

Damask and Callica Roses from a hundred year old garden, that we are offering under color this year, varieties still being in doubt. † FLESH. Double crean,y white tinted flesh pink, in clusters. †PINK. Several varieties. Shell pink and rose.

TRED. Very double dark red. Opens flat like quilled rosette. †YLLOW. Thorny canes, tiny leaves, double yellow roses.

†YORK AND LANCASTER. Pale red and white, striped pink.

Any of above 75 cents each; $\$ 7.50$ a dozen. 



\section{TEA ROSES}
$\dagger$ DUCHESS de BRABANT, pink.
†*LADY HILLINGDON, deep yellow.
†EUGENE MARLITT, red.
+SNOWFLAKE, pure white. †ETOILE d'LYON, yellow. $\uparrow$ MRS. B. R. CANT, rose. †THE BRIDE, white.
†WM. R. SMITH, flesh.

$\uparrow$ THE QUEEN, white.

Any of above $60 \mathrm{c} \in \mathrm{nts}$ each; three for $\$ 1.50$.

HYBRID TEA ROSES

Newer roses which have proved themselves in the garden.

$\dagger$ E. G. HILL. Large dark crimson $\ldots \ldots \ldots \ldots \ldots \ldots \ldots \ldots \ldots$

$\dagger^{*}$ JOANNA HILL. Creamy yellow flushed orange $\ldots \ldots \ldots \ldots 1.00$

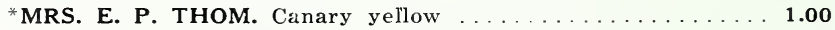

†*PRES. HOOVER. Cerise-pink, flame and yellow ....... 1.00

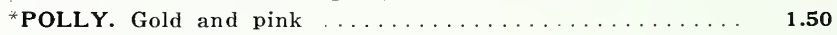

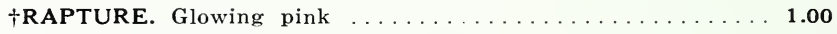

${ }^{*}$ SWANSDOWN. White shaded lemon .............. 1.50

$\dagger^{*}$ TALISMAN. Rich yellow, splashed orange-scarlet. . . . 1.00

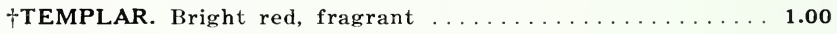

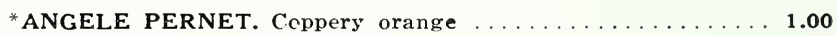

${ }^{*}$ CHAS. K. DOUGLAS. Crimson scarlet $\ldots \ldots \ldots \ldots \ldots \ldots \ldots . \mathbf{8 5}$

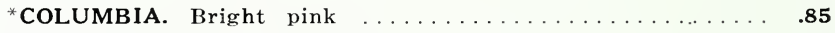

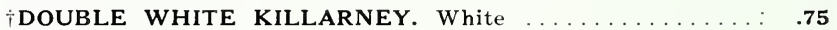

GEN. JACQUEMINOT (H. P.) Rich red . . . . . . . . .60

* JOHN RUSSELL. Immense black and crimson . . . . . . .85

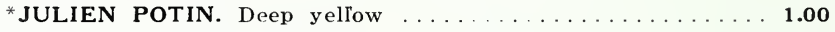

* J. OTTO THILOW. Immense glowing pink . . . . . . . 1.00

${ }^{*}$ KAISERIN AUgUSTA VIKTORIA. White $\ldots \ldots \ldots \ldots \ldots . .65$

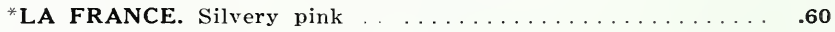

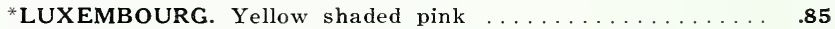

${ }^{*}$ MRS. WAKEFIELD CHRISTIE MILLER. Shell pink . . . . 1.00

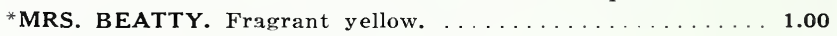

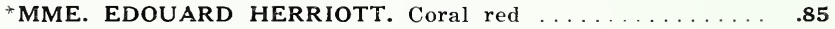

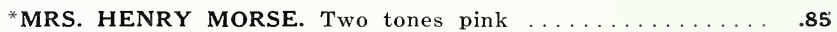

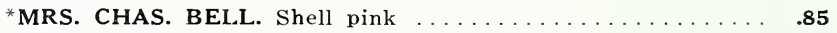

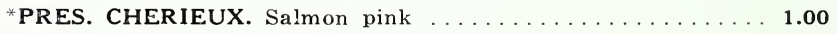

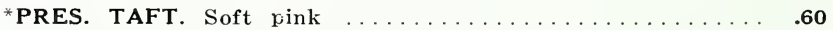

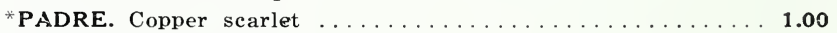

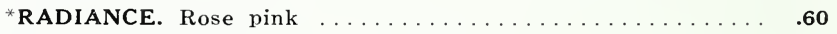

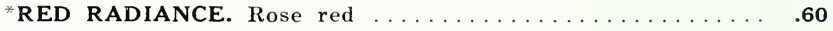

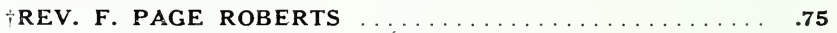

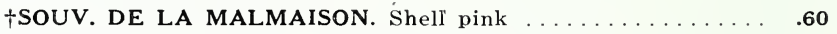

$\lceil$ CLOTHILDE SOUPERT. Clusters creamy pink .........60

$\dagger$ TIP TOP. Tiny pink and coppery yellow ............ .60

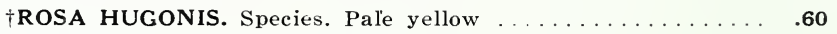

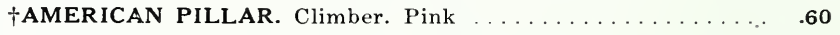

All roses are two year old, garden grown. †Own root stock only. *Budded stock only. $\dagger$ *Both own root and budded. 


\section{BUSINESS TERMS}

All Plants, Bulbs, etc. are forwarded by express, purchaser paying charges. Orders are dug and packed under our personal supervision, in rotation as received. Prices include packing and delivery to our nearest express office. We have no glass houses, our stock is hardy, garden grown.

In this list we are offering only a few of the perennials and rockplants we grow and can supply most of the standard sorts.

Orders accepted subject to stock on hand.

Due to causes over which we have no control, such as poor or improper soil, unfavorable weather, ignorant or careless culture, we can assume no responsibility for growth or productiveness of stock after it leaves our hands.

To find dozen rate multiply each price by ten.

Full remittance should accompany all orders. We do not send orders C.O.D. Do not send currency or silver except by registered letter. Remittances may be made by P. O. money crder, bank draft, or personal check made payable to Rosalie Dumble Davis.

Mail orders to:

\section{Rosalie M. Dumble Davis MOSS-SIDE 2535 MacGregor Way, Houston, Texas}

Iris and Narcissus Gardens at

GREENLANDS

ASHLANDS, VA. 
